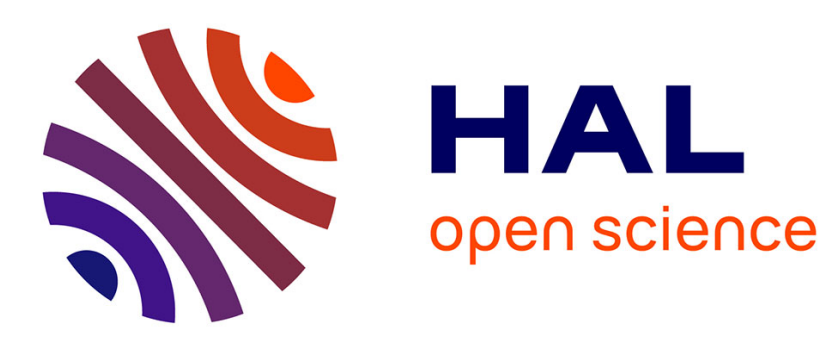

\title{
La disparition de Maurice Audin. Les historiens à l'épreuve d'une enquête impossible (1957-2014)
}

\author{
Sylvie Thénault
}

\section{To cite this version:}

Sylvie Thénault. La disparition de Maurice Audin. Les historiens à l'épreuve d'une enquête impossible (1957-2014). Histoire@Politique: revue du Centre d'histoire de Sciences Po, 2017, 31 (1), pp.140. 10.3917/hp.031.0140 . hal-02355614

\section{HAL Id: hal-02355614 https://hal.science/hal-02355614}

Submitted on 12 Nov 2019

HAL is a multi-disciplinary open access archive for the deposit and dissemination of scientific research documents, whether they are published or not. The documents may come from teaching and research institutions in France or abroad, or from public or private research centers.
L'archive ouverte pluridisciplinaire HAL, est destinée au dépôt et à la diffusion de documents scientifiques de niveau recherche, publiés ou non, émanant des établissements d'enseignement et de recherche français ou étrangers, des laboratoires publics ou privés. 


\title{
LA DISPARITION DE MAURICE AUDIN. LES HISTORIENS À L'ÉPREUVE D’UNE ENQUÊTE IMPOSSIBLE (1957-2014)
}

\author{
Sylvie Thénault
}

Centre d'histoire de Sciences Po | «Histoire@Politique »

2017/1 n $31 \mid$ pages 140 à 153

Article disponible en ligne à l'adresse :

https://www.cairn.info/revue-histoire-politique-2017-1-page-140.htm

Distribution électronique Cairn.info pour Centre d'histoire de Sciences Po.

(C) Centre d'histoire de Sciences Po. Tous droits réservés pour tous pays.

La reproduction ou représentation de cet article, notamment par photocopie, n'est autorisée que dans les limites des conditions générales d'utilisation du site ou, le cas échéant, des conditions générales de la licence souscrite par votre établissement. Toute autre reproduction ou représentation, en tout ou partie, sous quelque forme et de quelque manière que ce soit, est interdite sauf accord préalable et écrit de l'éditeur, en dehors des cas prévus par la législation en vigueur en France. Il est précisé que son stockage dans une base de données est également interdit. 
Sylvie Thénault, «La disparition de Maurice Audin. Les historiens à l'épreuve d'une enquête impossible (19572004) », Histoire@Politique, n³ 31, janvier-avril 2017 [en ligne, www.histoire-politique.fr]

\title{
La disparition de Maurice Audin. Les historiens à l'épreuve d'une enquête impossible (1957-2014)
}

\author{
Sylvie Thénault
}

« À partir du moment où le juge d'instruction n'avait pas fait son travail, c'était à l'historien de le faire1. » Ainsi Pierre Vidal-Naquet présentait-il son engagement dans l'affaire Audin, en pleine guerre d'indépendance algérienne (1954-1962). Militant du Parti communiste algérien (PCA), Maurice Audin avait été arrêté en 1957 à Alger, par les parachutistes. Ces derniers étaient alors titulaires des pouvoirs de police qui leur permettaient d'arrêter, de détenir et d'interroger tout individu. Le PCA, auquel Audin appartenait, était une des cibles de la répression française. Il avait été dissout et ses militants, entrés dans la clandestinité, s'étaient engagés dans la lutte pour l'indépendance, de diverses manières : maquis, propagande, terrorisme urbain ${ }^{2} . .$. Précisément, les parachutistes recherchaient activement les dirigeants du PCA et ils ont arrêté Audin en pensant qu'il pourrait les mener à eux ${ }^{3}$.

Audin n'est jamais réapparu. La recherche de la vérité, déclenchée par sa femme à Alger même, a été rapidement relayée en France par un Comité portant le nom du disparu. Sensibilisé parmi les premiers, Vidal-Naquet, spécialiste de la Grèce ancienne, est devenu l'éminent représentant du Comité et il a mis ses compétences d'historien au service de son combat. Il en a fait son premier ouvrage, L'Affaire Audin, publié par Jérôme Lindon, aux Éditions de Minuit, en 1958, puis réédité en 1989. Closes au pénal par un non-lieu en 1962, en raison de l'amnistie prononcée à la fin de la guerre, les procédures judiciaires ont été reprises au civil. J osette Audin demandait réparation du préjudice subi. In fine, le Conseil d'État l'a déboutée en 1978. Il a fallu une décision ministérielle, en 1983, pour que la femme de Maurice Audin et ses enfants obtiennent un dédommagement ${ }^{4}$.

L'histoire n'est pourtant pas close. Comme dans toutes les disparitions, l'absence de corps de la victime empêche d'y mettre un point final et rend impossible la cicatrisation des plaies de ceux que la disparition a fait souffrir ${ }^{5}$. La vérité reste

\footnotetext{
${ }^{1}$ Pierre Vidal-Naquet, L’Affaire Audin 1957-1978, Paris, Éditions de Minuit, 1989, p. 43.

2 Sur le PCA : Allison Drew, We are no Longer in France. Communists in Colonial Algeria, Manchester and New York, Manchester University Press, 2014. Pour un récit très factuel : Henri Alleg (dir.), La guerre d'Algérie, 3 tomes, Paris, Temps Actuels, 1981. Voir aussi le cas d'un militant en rupture avec la ligne du Parti : J ean-Luc Einaudi, Un Algérien, Maurice Laban, Paris, Le Cherche Midi Éditeur, 1999. Gilbert Meynier, enfin, aborde longuement la question des relations entre le FLN et le PCA dans Histoire intérieure du FLN, Paris, Fayard, 2002.

3 Merci à Nathalie Funès, François Demerliac, J osette Audin et Michèle Audin pour leurs relectures de cet article et leurs encouragements.

4 Pierre Vidal-Naquet, L'Affaire Audin..., op. cit., p. 186.

5 À ce sujet: Didier Bigo, « Disparitions, coercition et violence symbolique», Cultures \& Conflits [En ligne], 13-14 | printemps-été 1994, mis en ligne le 14 mars 2006, URL : http:// conflits.revues.org/ 181; DOI : 10.4000/conflits.181 [en ligne, consulté le 10 février 2017]. Sur les disparitions dues à l'armée française pendant la guerre d'indépendance algérienne, précisément : Raphaëlle Branche, La torture et
} 
Sylvie Thénault, «La disparition de Maurice Audin. Les historiens à l'épreuve d'une enquête impossible (19572004) », Histoire@Politique, n³ 31, janvier-avril 2017 [en ligne, www.histoire-politique.fr]

inconnue, faute de preuves. Dans ce contexte, de nouvelles révélations ont émergé dans les années 2011-2014, avec toutes les fragilités inhérentes à leur caractère tardif. Confrontées les unes aux autres, ainsi qu'aux déclarations de témoins retrouvés, elles donnent des versions des faits dissemblables, contrariant la recherche de la vérité. Ces discordances n'en intéressent pas moins les historiens, tant elles sont révélatrices de ce qui se joue dans ce processus de révélation si éloigné des faits : le rejeu de querelles anciennes, la volonté de se dédouaner de responsabilités culpabilisantes et criminelles - ou encore le désir de se mettre en avant, d'accéder à la notoriété médiatique et de provoquer la polémique.

Aujourd'hui comme pendant la guerre elle-même, par conséquent, cette affaire mobilise les historiens. Elle pose la question de leur apport, forts de leurs compétences et de leurs savoirs, à la recherche de la vérité. Si les historiens sont indéniablement des experts, de quelle plus-value peuvent-ils faire bénéficier l'enquête? Cet article, outre la démonstration de l'impossibilité pratique de faire émerger la vérité un demi-siècle après, livre un premier retour d'expérience.

\section{Du sort d'un homme à l'analyse d'un système. L'engagement de Pierre Vidal-Naquet}

Pierre Vidal-Naquet a revendiqué la spécificité de son engagement, en tant qu'historien. Se revendiquant d'une idée « ancienne, puisqu'elle remonte à Hérodote et à Thucydide », il pensait l'historien en « témoin de la vérité ${ }^{~}$ ». Aussi, poursuivaitil, «pendant la guerre d'Algérie, je n’ai pas écrit une seule ligne sans être convaincu de l'éminente dignité de ma profession ${ }^{7}$ ». Plus près de lui qu'Hérodote et Thucydide, J ean J aurès lui servait de modèle. Il se référait tout particulièrement aux Preuves, dans lesquelles J ean Jaurès examinait les pièces produites par l'accusation du capitaine Dreyfus, pour en démontrer la fausseté8.

De même, Vidal-Naquet a minutieusement examiné les papiers fournis par les autorités françaises après la disparition d'Audin. Alors que des réponses dilatoires étaient d'ordinaire opposées aux familles demandant des nouvelles d'un des leurs, arrêté par les parachutistes et qui n'avait pas reparu depuis, les militaires sont allés, dans le cas d'Audin, jusqu'à inventer et jouer un scénario. D’après eux, Audin aurait été placé à l'arrière d'une jeep pour être transféré du lieu où il était détenu et il aurait profité d'un virage un peu serré pour sauter de la voiture puis s'enfuir. Après que le chauffeur, le sergent Cuomo, a stoppé le véhicule, son passager, le sergent Misiri, armé, a tiré dans la direction du pseudo-Audin mais sans pouvoir l'atteindre. Un riverain, sorti de chez lui, pouvait en témoigner. Pièce maîtresse de la version officielle, les militaires ont produit un compte rendu d'évasion. Le sergent Misiri, tenu pour responsable de l'évasion, a même été puni pour sa négligence.

l'armée, Paris, Gallimard, 2001, p. 137-146. L'historiographie s'est en outre emparée des disparitions de Français à la fin du conflit. Tout en ayant les mêmes effets sur les proches des victimes, elles n'ont pas eu la même ampleur et ne relèvent pas de la même analyse dans la mesure où il ne s'agit pas de disparitions dues à des forces étatiques agissant dans le cadre d'une politique répressive : J ean-J acques Jordi, Un silence d'État. Les disparus civils européens de la guerre d'Algérie, Paris, Éditions SOTECA, 2011.

6 Pierre Vidal-Naquet, Face à la raison d’État. Un historien dans la guerre d'Algérie, Paris, La Découverte, 1989, p. 8.

7 Ibid.

8 Ibid., p. 10. Jean Jaurès, Les Preuves. Affaire Dreyfus (29 septembre 1898), Paris, La Petite République, 1898. 
Sylvie Thénault, «La disparition de Maurice Audin. Les historiens à l'épreuve d'une enquête impossible (19572004) », Histoire@Politique, n³ 31, janvier-avril 2017 [en ligne, www.histoire-politique.fr]

J osette Audin déposant plainte contreX pour homicide volontaire, l'instruction a collecté ces premiers papiers avant d'en produire elle-même de multiples autres, dont des procès-verbaux d'interrogatoire. C'est ce dossier que Vidal-Naquet a passé au crible dans L'Affaire Audin, avec sa lecture d'historien attentif à la reconstitution minutieuse des faits. Relevant de multiples incohérences, au premier rang desquelles les horaires du trajet de la jeep, il démontrait le caractère mensonger de cette version. $\mathrm{Si}$ la démonstration était la sienne, la mise en forme du manuscrit, devait tout à son éditeur, J érôme Lindon, au point que Vidal-Naquet a par la suite estimé qu'il aurait dû signer l'ouvrage'?

L'instruction de la plainte déposée par J osette Audin à Alger a ensuite été transférée au Parquet de Rennes, dans une logique de dépaysement des affaires de torture d'Algérie en métropole qui s'est progressivement imposé au ministère de la J ustice ${ }^{10}$. Le juge d'instruction de Rennes a repris l'affaire et convoqué nombre de témoins ; en vain. C'est cette instruction qui a été close par un non-lieu en 1962. Réciproque, l'amnistie impliquait en effet la libération des personnes ayant combattu côté algérien pour l'indépendance, tandis qu'elle exonérait de toute poursuite les membres des forces de l'ordre françaises qui se seraient rendus coupables de crimes dans l'exercice de leurs fonctions ${ }^{11}$.

L'expertise des pièces du dossier d'instruction impliquait de reconstituer, concrètement, au plus près de la réalité du terrain, dans le quotidien, le travail des militaires quadrillant la ville. Aussi Vidal-Naquet a-t-il cherché à comprendre le fonctionnement du système répressif mis en place à Alger par les parachutistes, au point d'en devenir, in fine, le premier historien. Si ce système est aujourd'hui connu, l'analyser à l'époque n'avait rien d'évident ${ }^{12}$. Non seulement il était alors inédit mais les militaires entouraient leurs opérations du plus grand secret.

Les ouvrages de Vidal-Naquet témoignent de son évolution. En 1962, il signait La Raison d'Etat, un recueil de documents collectés par le Comité Maurice Audin, sur la pratique de la torture, au fur et à mesure de la guerre. Il les présentait un à un, les annotait et leur adjoignait des déclarations de responsables politiques susceptibles de les éclairer. Il les encadrait également par une introduction et une conclusion d'ensemble. Il s'y livrait à un exercice ordinaire d'historien : l'édition critique de documents. Il ne cherchait pas à établir la vérité de la pratique de la torture, qui était connue et ne pouvait être niée. Son ambition était de démontrer que cette pratique était « une affaire qui nous concerne tous ${ }^{13} »$. En effet, argumentait-il :

« Comment déterminer le rôle dans l'État futur de la magistrature, de l'armée ou de la police si nous ne savons pas d'abord comment l'État en tant que tel s'est comporté devant les problèmes posés par la répression de l'insurrection algérienne, comment il a été informé par eux dont c'était la mission d'informer, comment il a réagi en présence de ces informations, comment il en a informé à son tour les citoyens...L'État,

\footnotetext{
9 Pierre Vidal-Naquet, Mémoires. Le trouble et la lumière 1955-1998, Paris, Seuil/ La Découverte, 1998, p. 71.

10 À ce sujet : Sylvie Thénault, Une drôle de justice. Les magistrats dans la guerre d’Algérie, Paris, La Découverte, 2001.

${ }^{11}$ Stéphane Gacon, L’amnistie. De la Commune à la guerre d’Algérie, Paris, Le Seuil, 2002.

${ }^{12}$ Ce système répressif a été analysé par Raphaëlle Branche dans La torture et l'armée..., op. cit., ainsi que par Gilbert Meynier dans Histoire intérieure du FLN..., op. cit.. La journaliste Marie-Monique Robin l'a également décrit sur le fondement de témoignages : Escadrons de la mort. École française, Paris, La Découverte, 2008.

13 Pierre Vidal-Naquet, La raison d’État, Paris, La Découverte, 2002 (rééd.), p. 17.
} 
Sylvie Thénault, «La disparition de Maurice Audin. Les historiens à l'épreuve d'une enquête impossible (19572004) », Histoire@Politique, n³ 31, janvier-avril 2017 [en ligne, www.histoire-politique.fr]

c'est-à-dire l'armée, c'est-à-dire la police, c'est-à-dire l'administration, c'est-à-dire la justice ${ }^{14}$. »

À terme, Vidal-Naquet a produit un ouvrage devenu incontournable : La torture dans la République, paru aux Éditions de Minuit en 1972. Ce livre a d'abord été publié en anglais et en italien, dès 1963, à la demande d'un éditeur étranger ${ }^{15}$. Il s'agissait cette fois d'un essai. Dans la lignée de La raison d’État, Vidal-Naquet y défendait fortement sa thèse : celle de la contamination progressive de l'État par la torture. La démonstration n'en était pas moins dotée d'un fondement empirique. Les chapitres reposaient sur quantité de documents et d'informations collectées à la faveur du combat mené contre la torture pendant la guerre. « Ce livre n'est pas un ouvrage d'érudition, s'expliquait Vidal-Naquet, mais j'aimerais que le lecteur sache qu'il repose sur une documentation solide et de première main ${ }^{16}$. »

En pionnier, Vidal-Naquet a mobilisé ses compétences d'historien dans un mouvement d'élargissement progressif, à partir de l'étude de pièces produites dans un cas pour aboutir à une analyse d'ensemble, en passant par l'édition critique de documents. Un tel engrenage s'inscrit d'évidence dans la temporalité même du conflit. Pour Vidal-Naquet, pris dans l'urgence du moment, la dénonciation de la torture impliquait, en historien, d'en comprendre les ressorts ainsi que, en citoyen, d'alerter ses contemporains sur la menace qu'elle représentait pour les libertés et la nature même de l'État. Plus d'un demi-siècle après les faits, alors qu'une historiographie s'est constituée et que les archives sont en grande partie inventoriées et accessibles, quelle pouvait être la contribution d'un historien à l'enquête qui prenait une nouvelle impulsion?

\section{Le manuscrit d'Yves Godard ou l'ex-OAS contre de Gaulle?}

Dans la première édition de L'Affaire Audin, rédigée à chaud, en 1957-1958, alors que l'instruction traînait encore à Alger, Vidal-Naquet a formulé l'hypothèse qu'Audin était décédé au cours de son interrogatoire sous la torture, par " accident ${ }^{17}$ ». Par la suite, il a été convaincu que le lieutenant Charbonnier, l'officier de renseignements qui avait torturé Audin, l'avait étranglé lors d'un accès de fureur. L'armée protégeant les siens et les témoins se dérobant, cependant, aucune preuve n'a pu être apportée. L'information repose sur le témoignage d'un officier de police détaché à l'État-Major du secteur Alger-Sahel, le secteur dans lequel s'est produite la disparition d'Audin. Ce policier a cependant refusé de confirmer cette version par écrit devant le juge d'instruction de Rennes ${ }^{18}$. Il n'en a pas non plus donné le fondement: a-t-il simplement relayé ce qu'il a entendu dire à l'État-Major ou avait-il des éléments plus solides en sa possession?

À l'automne 2011, une journaliste au Nouvel Observateur, Nathalie Funès, a contacté la famille - J osette et ses enfants, Michèle et Pierre - pour leur montrer un document

\footnotetext{
14 Pierre Vidal-Naquet, La raison d'État..., op. cit., p. 17.

15 Torture: a Cancer of Democracy, Harmondsworth, Penguin Books, 1963 ; Lo Stato di Tortura, Bari, Laterza, 1963.

16 Pierre Vidal-Naquet, La torture dans la république, op. cit., p. 5.

${ }^{17}$ Le mot est entre guillemets dans le texte de L'Affaire Audin publié en 1958 et repris dans l'édition de 1989, p. 106.

18 Pierre Vidal-Naquet, L'Affaire Audin..., op. cit., p. 145.
} 
Sylvie Thénault, «La disparition de Maurice Audin. Les historiens à l'épreuve d'une enquête impossible (19572004) », Histoire@Politique, n³ 31, janvier-avril 2017 [en ligne, www.histoire-politique.fr]

nouveau. Au hasard d'une enquête portant sur un autre sujet, elle avait été amenée à découvrir un manuscrit de l'ex-colonel Godard, dans lequel celui-ci donnait une nouvelle version de ce qui serait arrivé à Audin. Ici, l'expertise historienne consistait à évaluer la crédibilité du contenu du manuscrit à l'aune de l'état des savoirs sur la guerre d'indépendance algérienne. Il fallait, tout à la fois, resituer le colonel Godard dans l'organigramme militaire d'Alger en 1957, revenir sur sa biographie, éclairer du mieux possible - les circonstances de la rédaction de son texte et tenter de restituer l'histoire même de ce dernier. Les enjeux de la révélation de Godard, qui aurait dû intervenir dans les années 1970, sont alors apparus comme capitaux.

$\mathrm{Au}$ moment de l'arrestation d'Audin, le colonel Godard commandait le secteur militaire concerné : le secteur Alger-Sahel. Devenu ensuite chef de la Sûreté nationale en Algérie, Godard a fini par rejoindre l'OAS (Organisation Armée Secrète), cette organisation terroriste qui combattait l'indépendance de l'Algérie et prenait tout particulièrement le général de Gaulle pour cible. Après l'indépendance, Godard a vécu dans la clandestinité afin d'échapper aux sentences prononcées contre lui par contumace - il avait été condamné à mort. Il aurait pu revenir en France après l'amnistie bénéficiant aux membres de l'OAS, en 1968, mais il a fini sa vie en Belgique, où il est décédé en $1975^{19}$.

Godard avait entrepris de rédiger sa propre histoire de la « Bataille d'Alger » - pour reprendre l'expression consacrée en dépit de ses imperfections. Elle est trop normative, en effet, pour décrire ce qu'a été l'engagement des parachutistes contre le FLN dans la ville, en 1957, conduisant à un quadrillage serré de l'espace urbain, des arrestations en masse et la généralisation de la torture ${ }^{20}$. L'histoire que Godard comptait en écrire devait comprendre trois tomes. Le premier est sorti en 1972, aux éditions Fayard ${ }^{21}$. Le récit s'arrêtait cependant avant l'arrestation d'Audin. Le mois de juin était traité dans le second tome, rédigé, mais qui n’a jamais été publié pour des raisons encore inconnues - l'état de santé de Godard a été invoqué22. C'est ce manuscrit que Nathalie Funès a retrouvé. Son existence n'était pas secrète. Un extrait en a été publié en 1979 dans un ouvrage dirigé par François Porteu de la Morandière, président de l'Union nationale des Combattants en Afrique du Nord (UNAC), une association qui a depuis intégré l'Union nationale des combattants (UNC) ${ }^{23}$. L'UNC se situe clairement à droite de l'échiquier politique. Elle a en particulier combattu le choix du 19 mars comme date commémorative de la guerre d'indépendance algérienne $^{24}$. François Porteu de la Morandière a également été dirigeant et élu du Front national dans la seconde moitié des années 198025. Nathalie Funès a d'ailleurs

\footnotetext{
19 Sur l'OAS et le rôle de Godard en son sein : Olivier Dard, Voyage au cour de l'OAS, Paris, Perrin, 2005; Anne-Marie Duranton-Crabol, L'OAS, la peur, la violence, Bruxelles, André Versaille éditeur, 2012.

20 De façon significative, Gilbert Meynier parle de «la grande répression d'Alger », dans Histoire intérieure..., op. cit., p. 229.

${ }^{21}$ Les Trois batailles d'Alger, tome 1: Les paras dans la ville, Paris, Fayard, 1972.

22 Dans François Porteu de la Morandière, Soldats du djebel. Histoire de la guerre d'Algérie, Ivry, Société de Production Littéraire, 1979, p. 124.

${ }^{23}$ François Porteu de la Morandière, Soldats du djebel..., op. cit.. L'extrait du manuscrit d'Yves Godard occupe les pages 124 à 126.

24 Voir l'édito du président de l’UNC, consacré à la commémoration du 19 mars, dans son Bulletin, La Voix du combattant en mars 2016 (p. 3) : http:// www.unc.fr/journal/la-voix-du-combattant [en ligne, consultéle 15 février 2017].

25 Sur François Porteu de la Morandière, voir Guy Birenbaum et Bastien François, « Unité et diversité des dirigeants frontistes », dans Nonna Mayer et Pascal Perrineau (dir.), Le Front national à découvert, Paris, Presses de Sciences Po, 1996, p. 83-106. Pour un aperçu des idées de François Porteu de la
} 
Sylvie Thénault, «La disparition de Maurice Audin. Les historiens à l'épreuve d'une enquête impossible (19572004) », Histoire@Politique, n³ 31, janvier-avril 2017 [en ligne, www.histoire-politique.fr]

eu connaissance de l'existence du manuscrit en enquêtant dans ces milieux. Si, en tant que journaliste, elle ne peut que protéger sa source, l'information est en ellemême significative. Il est certes impossible de reconstituer précisément la circulation du manuscrit et/ ou de son contenu depuis la publication d'extraits en 1979 dans le livre dirigé par François Porteu de la Morandière jusqu'à la transmission de l'information à Nathalie Funès en 2011. Reste que le manuscrit - ou son texte ? - a navigué dans des courants politiques situés à l'extrême droite et soudés par l'antigaullisme après l'indépendance de l'Algérie ${ }^{26}$.

L'authenticité du texte ne fait pas de doute. Nathalie Funès a retrouvé l'original dans les archives privées de l'ex-colonel, à Stanford ${ }^{27}$. Godard y prétendait qu'Audin avait été exécuté par un commando « assisté du commandant Aussaresses ${ }^{28}$ ». Officier de renseignements, Aussaresses dirigeait, à Alger, en 1957, une équipe notamment chargée de l'exécution de prisonniers importants arrêtés par les parachutistes et qu'il était décidé d'éliminer ${ }^{29}$. D’après le récit de Godard, Audin aurait été exécuté par erreur. Audin n'était pas, en effet, un prisonnier important, dans la mesure où il n'était pas un responsable du PCA. Les parachutistes l'avaient arrêté en pensant qu'il serait susceptible de les mener à Henri Alleg, l'un des principaux dirigeants du PCA. Or Alleg avait été arrêté peu après Audin et il était détenu au même endroit que lui. D'après Godard, c'est Alleg que devait assassiner le commando d'Aussaresses mais il serait trompé de prisonnier. Godard allait même jusqu'à donner le nom de l'exécuteur : Gérard Garcet.

$\mathrm{Au}$ vu de l'historiographie de la guerre d'indépendance algérienne - et en particulier de la connaissance des milieux militaires et du système répressif algérois en 1957 - , il est possible que le colonel Godard ait menti, entièrement ou en partie. L'hypothèse même d'une exécution par un commando est à vérifier. Qu'une telle exécution ait résulté d'une erreur suppose en outre que les militaires aient confondu Audin et Alleg, ce qui n'est pas très plausible. Le signalement d'Henri Alleg, recherché, était connu et il différait largement du physique de Maurice Audin. Enfin, lorsqu'il désignait Garcet comme l'assassin d'Audin, Godard prenait soin d'indiquer que Garcet était l'aide de camp du général Massu - son ancien aide de camp, en réalitéet il lui faisait porter la responsabilité de l'exécution, avec Aussaresses. L'inimitié de Godard pour Aussaresses était notoire et Aussaresses a dit ensuite qu'elle était réciproque. Non seulement Godard aurait désapprouvé les exécutions que pratiquaient les commandos d'Aussaresses à Alger, en 1957, mais, avant même l'Algérie, les deux hommes avaient été professionnellement en concurrence et en désaccord ${ }^{30}$.

Morandière, voir son ouvrage : Sacrée Marianne ! Fausse crise politique et vraie crise morale, Issy-lesMoulineaux, Muller édition, 2000.

26 A ce sujet : Ariane Chebel d'Appollonia, L'extrême droite en France. De Maurras à Le Pen, Bruxelles, Complexe, 1987; Valérie Igounet, Le Front national de 1972 à nos jours, Paris, Seuil, 2014.

${ }^{27}$ Les archives d'Yves Godard sont conservées à la Hoover Institution on War, Revolution and Peace, à l'université de Stanford : http:// www.oac.cdlib.org/ findaid/ ark:/ 13030/tf387002s7/ ?query=godard. [en ligne, consulté le 8 février 2017]. Des copies des documents peuvent être envoyées sur demande.

28 On peut lire des reproductions de l'extrait du manuscrit d'Yves Godard sur le site de la LDH de Toulon, à cette adresse : http://ldh-toulon.net/affaire-Audin-la-verite-doit-etre.html [en ligne, consulté le 8 février 2017]

${ }^{29}$ Les révélations de Paul Aussaresses sur cette activité ont fait sensation en 2001 : Services spéciaux. Algérie, 1955-1957, Paris, Perrin, 2001.

30 Quelques années après la guerre, Yves Courrière mentionnait déjà l'animosité de Godard envers Aussaresses: La guerre d’Algérie, tome 2 : Le temps des léopards, Paris, Fayard, 1969, p. 531-532. Aussaresses est revenu sur leurs relations antérieures dans Services Spéciaux..., op. cit., p. 92-93. 
Sylvie Thénault, «La disparition de Maurice Audin. Les historiens à l'épreuve d'une enquête impossible (19572004) », Histoire@Politique, n³ 31, janvier-avril 2017 [en ligne, www.histoire-politique.fr]

À travers Garcet, présenté comme l'aide de camp de Massu, Godard impliquait aussi ce dernier, avec lequel il avait eu des différends. Godard critiquait en effet la mission de police acceptée par Massu à Alger en 1957 tandis que Massu le jugeait peu efficace $^{31}$. Au-delà, Massu était une cible politique dans les cercles que fréquentait l'ex-colonel dans le contexte post-Mai 68, au moment où il rédigeait son manuscrit. Massu incarnait en effet le loyalisme envers de Gaulle, au contraire du choix fait par Godard. Non seulement, pendant la guerre d'indépendance algérienne, en dépit de ses désaccords avec la politique du chef de l'État, Massu n'a jamais rejoint ni soutenu l'OAS, mais en outre, en mai 68, c'est vers lui que de Gaulle s'est tourné en allant le rencontrer secrètement à Baden-Baden. Ainsi la fidélité de Massu à de Gaulle a-t-elle été réaffirmée, alors que le chef de l'État restait honni des anciens partisans de l'Algérie française ${ }^{32}$. La loi d'amnistie que de Gaulle a fait voter en juillet 1968, au bénéfice des derniers condamnés de l'OAS, n'a en rien contribué à infléchir les positions des ex-militants de l'Organisation à l'égard du chef de l'État. Cette loi visait en fait l'électorat d'extrême droite qu'il s'agissait de rallier dans la perspective du référendum sur des réformes institutionnelles, conçues pour sortir de la crise référendum à l'issue duquel, in fine, de Gaulle, désavoué par un résultat négatif, a quittéle pouvoir ${ }^{33}$.

L'expertise du contenu du manuscrit de Godard conduit à revenir sur le récit de la guerre d'indépendance algérienne, tel qu'il s'est constitué dans le contexte des années 1970 parmi des anciens d'Algérie se retrouvant à l'extrême droite. Elle conduit également à une immersion dans le milieu du commandement algérois de l'année 1957, révélant des tensions de diverses natures - personnelles, professionnelles, tactiques - entre ses membres. Apparait aussi nettement l'impact de la disparition d'Audin au sein du commandement algérois. Elle semble y avoir constitué un événement, commenté et avivant des tensions préexistantes. Le policier défendant la thèse de l'étranglement par Charbonnier, d'ailleurs, était lui-même en détachement à l'État-major du secteur Alger-Sahel. Les témoignages de militaires sont au cœeur de l'enquête ; elle y a trouvé ses limites.

\section{Aux limites de l'enquête : les témoignages de militaires sur la disparition d'Audin}

Une exploration de la bibliographie révèle que la version de la mort d'Audin donnée par Godard dans son manuscrit avait déjà été publiée. Elle figure en effet dans la vaste fresque en quatre tomes consacrée à La guerre d'Algérie par le journaliste Yves Courrière, parue entre 1968 et 1972 chez Fayard - le même éditeur que celui du premier tome de Godard. Cette histoire était rédigée dans un style vivant, imagé et romancé qui a contribué à son succès public ${ }^{34}$. Pour les historiens, l'ouvrage reste délicat à manier. Non seulement le primat donné au style conduit l'auteur à déformer les faits mais aucune information n'est référencée. La liste des témoins donnée en

\footnotetext{
${ }^{31}$ Yves Godard, Les paras..., op. cit., p. 228-229 et J acques Massu, La vraie bataille d'Alger, Paris, Plon, 1971, p. 108-109.

32 Massu en a témoigné dans un livre au titre significatif : Baden 68. Souvenirs d'une fidélité gaulliste, Paris, Plon, 1983. Pour un aperçu synthétique de la politique gaulliste en 1968 : Boris Gobille, Mai 68, Paris, La Découverte, 2008.

33 Stéphane Gacon, L'amnistie.., op. cit. Olivier Dard analyse également «le Mai 68 de l'Algérie française », dans Voyage au cœur del'OAS, op. cit.

34 Yves Courrière en a témoigné dans ses Mémoires : Éclats de vie, Paris, Fayard, 2003, p. 387-420.
} 
Sylvie Thénault, «La disparition de Maurice Audin. Les historiens à l'épreuve d'une enquête impossible (19572004) », Histoire@Politique, n³ 31, janvier-avril 2017 [en ligne, www.histoire-politique.fr]

annexe n'identifie en outre qu'une partie d'entre eux. Dans le passage sur la mort d'Audin, Courrière rapportait que « tout le monde [savait] qu'O... [était] responsable de la mort d'Audin. Ou plutôt ses hommes. Car il [s'agissait] d'une méprise ${ }^{35} »$. D'une manière significative de sa façon d'écrire l'histoire, Courrière allait jusqu'à imaginer la scène d'exécution et mettre ces mots dans la bouche d'Audin : «Vous faites erreur, je suis européen ${ }^{36} . . . »$ Cette version est celle donnée dans le manuscrit de Godard, dont le nom est cité par Courrière dans son ouvrage. Godard en serait donc la source. Courrière, cependant, aurait-il aussi rencontré Aussaresses, que désignait la lettre « $\mathrm{O}$ » ? Rien ne permet de le savoir. Courrière est resté allusif sur ses sources dans ses mémoires et ni les éditions Fayard, ni sa veuve, interrogés, n’ont pu éclairer la question ${ }^{37}$.

$\mathrm{Au}$ contraire de Godard, toutefois, Courrière ne citait pas le nom de Garcet et invoquait la responsabilité d'Aussaresses, même s'il renvoyait également à ses « hommes ». Garcet était d'évidence un témoin-clé. Nathalie Funès a réussi à le retrouver ainsi que d'autres protagonistes ${ }^{38}$. La quête de la vérité a en outre été menée par François Demerliac, un documentariste qui a réalisé un film sur l'affaire Audin $^{39}$. Tous deux ont recherché et contacté divers témoins de cette histoire, dialogué au moins par téléphone avec ceux qui ne voulaient pas aller plus loin, rencontré et éventuellement enregistré - voire filmé pour François Demerliac - ceux qui le voulaient bien. Semant le doute, certains s'en tenaient à de strictes dénégations qui ne semblaient pas toujours dénuées de crédibilité, d'autres mentaient de manière éhontée, d'autres encore racontaient tout et son contraire, parfois au cours d'un seul et même entretien. Peu se sont dérobés à tout contact, à l'exception notable d'Aussaresses.

Bien qu'ayant accompagné leurs recherches, il ne m'est pas possible de livrer ici le contenu de tels échanges. Non seulement le format d'un article n'y suffirait pas mais ces contacts et entretiens n'ont pas donné lieu à la production de témoignages respectant le cadre formalisé de la pratique historienne: enregistrement systématique, retranscription, soumission au témoin pour accord, engagement éventuel d'anonymat pour toute citation... D'évidence, cette méthode aurait été contre-productive. Dans le contexte de la recherche de la vérité sur la disparition d'Audin, les pratiques journalistiques sont plus adaptées. Il faut passer par une phase d'enquête souterraine, multipliant les contacts, recueillant des informations tous azimuts, parfois de façon détournée, hors de tout dispositif risquant d'alerter et/ ou d'effrayer des témoins restés sur leurs gardes, avant de procéder à un travail de recoupement permettant d'approcher au mieux la vérité. La restitution même du résultat peut se faire sous l'empire de la protection des sources, si celle-ci est posée d'emblée comme condition du recueil de l'information. L'administration de la preuve doit alors passer par d'autres moyens que la référence à un témoignage pouvant être rendu public.

L'enquête n'a toutefois pas atteint ce stade ultime. La difficulté reste de démêler le vrai du faux, notamment en cernant les enjeux contemporains des dires des uns et

\footnotetext{
35 Yves Courrière, La guerre d’Algérie, tome 2, op. cit., p. 532.

36 Ibid.

${ }^{37}$ Yves Courrière, Éclats..., op. cit., p. 387-420.

38 Nathalie Funès a rendu compte de son contact avec Gérard Garcet: « Révélations sur l'Affaire Audin », dans Le Nouvel Observateur, 1er mars 2012, p. 10-14.

39 Diffusé sur Public Sénat en 2010, le film est aujourd'hui disponible en DVD : Maurice Audin, La disparition, Éditions Montparnasse, 2010.
} 
Sylvie Thénault, «La disparition de Maurice Audin. Les historiens à l'épreuve d'une enquête impossible (19572004) », Histoire@Politique, n³1, janvier-avril 2017 [en ligne, www.histoire-politique.fr]

des autres. Le cas de Cuomo, le chauffeur de la jeep, ancien adjudant-chef et moniteur au 1er régiment de chasseurs parachutistes, qui s'est exprimé publiquement en 2001, l'illustre parfaitement ${ }^{40}$. En mai 2001, en effet, Aussaresses a publié un livre au beau milieu d'un véritable débat national sur la torture et les exécutions sommaires en Algérie pendant la guerre d'indépendance ${ }^{41}$. Il affirmait que Cuomo avait fait partie d'une équipe spéciale, clandestine, à son service ${ }^{42}$. Cuomo s'en est alors défendu dans La République des Pyrénées ${ }^{43}$. Interrogé sur la disparition d'Audin, il déclarait que l'homme transporté à l'arrière de la jeep était cagoulé. Fait surprenant - et qui rend la déclaration suspecte - , jamais cette cagoule n'avait été mentionnée auparavant. Cuomo fragilisait évidemment la version officielle. Si le soidisant prisonnier était cagoulé, comment prouver qu'il s'agissait d'Audin ? D'un point de vue personnel, Cuomo se dédouanait de toute responsabilité - si le prisonnier était cagoulé, Cuomo ne pouvait plus rien attester. Il affirmait même être prêt à rencontrer J osette Audin et n'avoir rien à craindre. Paradoxalement, il était sorti de sa réserve pour se protéger. Aussaresses, en revanche, très visiblement en quête de notoriété, s'érigeait en figure centrale dans la recherche de la vérité.

Aussaresses n'a pourtant pratiquement rien dit de la disparition d'Audin dans Services spéciaux, en 2001, et il a refusé de se livrer au cours d'entretiens avec J eanCharles Deniau, parus en 2008 aux éditions du Rocher : Je n'ai pas tout dit. Les confidences qu'il a finies par faire à cejournaliste ont été publiées de façon posthume. Ainsi est sorti, en janvier 2014, un livre au titre retentissant : La vérité sur le mort de Maurice Audin, aux Éditions des Équateurs. J ean-Charles Deniau y reprenait la thèse de l'exécution d'Audin par un commando aux ordres d'Aussaresses, comprenant Garcet, mais non par erreur : il se serait agi de faire un exemple. L'exécution aurait servi d'avertissement à tous ceux qui auraient été tentés de s'engager pour l'indépendance. Ce motif est plus qu'étonnant. Outre qu'il est invraisemblable qu'une exécution ait valeur d'exemple si elle ne s'accompagne pas de publicité, les disparitions d'individus arrêtés par l'armée étaient suffisamment nombreuses et connues à Alger en 1957 pour que les partisans de l'indépendance algérienne aient été conscients des risques qu'ils encouraient. Nul besoin d'exécuter Audin pour les alerter. Les fondements du livre de Jean-Charles Deniau sont en outre fragiles: parole d'Aussaresses et déclarations de Pierre Misiri, le passager de la jeep, auteur des coups de feu lors de la pseudo-évasion - le témoignage de ce dernier mériterait pourtant d'être considéré avec les plus grandes précautions. Pour affirmer qu'il livrait la « vérité », enfin, J ean-Charles Deniau a gommé tout ce qui pouvait semer le doute. Il n'étudie aucune autre des hypothèses existantes: un étranglement par Charbonnier, une exécution par erreur ou encore - pourquoi pas - la mort sous la torture. Ce livre ne peut que susciter la suspicion. Il a été très vivement rejeté par Josette Audin et ses enfants ${ }^{44}$. L'élucidation du sort d'Audin s'avère en l'état impossible.

40 Son décès, le 1er janvier 2017, a été annoncé par l'Amicale du régiment: http://www.campsparachutistes.org/t6164-deces-de-l-adc-yves-cuomo [n ligne : consulté le 30 mars 2017].

41 Pour une fine chronologie et une analyse de ce débat: Tramor Quémeneur, « La mémoire mise à la question : le débat sur les tortures dans la guerre d'Algérie, juin 2000-septembre 2001 », Regards sur l'actualité, n² 276, décembre 2001, p. 29-40.

42 Services spéciaux..., op. cit., p. 113.

43 La République des Pyrénées, 11 mai 2001, p. 1 et 3.

44 On peut écouter la réaction de J osette Audin à la Matinale de France Inter le 8 janvier 2014 : https:// www.franceinter.fr/emissions/le-7-9/le-7-9-08-janvier-2014 [en ligne: consulté le 22 février 2017]. 
Sylvie Thénault, «La disparition de Maurice Audin. Les historiens à l'épreuve d'une enquête impossible (19572004) », Histoire@Politique, n³ 31, janvier-avril 2017 [en ligne, www.histoire-politique.fr]

\section{Savoirs et compétences des historiens : quelle plus-value dans l'enquête?}

Cette enquête pose la question des frontières séparant les historiens - et, plus généralement, les chercheurs en sciences humaines et sociales - de professionnels de l'investigation, journalistes et documentaristes en l'occurrence. Dans ce cas, ce ne sont pas une distance ni des différences et encore moins des désaccords qui sont apparus. Bien au contraire, l'enquête a pu être menée de pair et dans l'entente, sur un socle commun de principes et de méthodologie, au premier rang desquels le recoupement des sources et la prudence: impossible de défendre une thèse au détriment d'une autre, en l'absence de preuve. L'accord a aussi régné sur la relation avec la famille à qui cette histoire appartient et qui doit être associée aux démarches accomplies. Loin de faciliter alors la définition de règles qui distingueraient strictement les historiens d'autres enquêteurs, ce travail a estompé les frontières entre les pratiques professionnelles des uns et des autres. S'est dessiné un espace de collaboration, chacun apportant ses compétences et ses savoirs à une entreprise qui, si elle n'est pas totalement commune, est partagée. Il en découle une certitude : celle de la proximité, dans ce cas précis, du travail d'investigation mené par une journaliste, un documentariste et une historienne. Ces trois professions partagent en outre une limite forte dans la recherche de la vérité et l'administration de la preuve : l'absence de moyens scientifiques d'investigation qui finissent par être indispensables ${ }^{45}$. Précisément, dans l'enquête sur la disparition d'Audin, une expertise génétique pourrait être un jour sollicitée, si des restes étaient retrouvés. J ean-Charles Deniau prétend avoir localisé le lieu d'exécution et d'inhumation de Maurice Audin ${ }^{46}$. Le cas échéant, il appartiendrait à la famille de décider de telles expertises.

Quelle peut être dès lors la spécificité de l'intervention des historiens? La connaissance du contexte factuel, celle de la bibliographie et de l'historiographie sont au fondement de leur expertise. L'évolution de la connaissance historique depuis l'époque de la guerre elle-même, lorsque Vidal-Naquet cherchait en pionnier à comprendre le système répressif mis en place à Alger, est fondamentale. Aujourd'hui, ce système est parfaitement analysé. De ce point de vue, l'effet du temps écoulé depuis les faits est très exactement contraire à celui qu'il produit sur une enquête portant sur un cas précis. Comme le démontre le cas de la disparition de Maurice Audin, le temps écoulé depuis les faits est un obstacle difficile à surmonter lorsqu'il s'agit de rechercher la vérité sur le sort d'un individu. Au contraire, l'analyse du système répressif dans sa globalité gagne en intelligibilité et celle-ci facilite l'évaluation des informations recueillies.

La pratique des archives est cependant l'apport le plus spécifique des historiens. En 2012, J osette Audin a obtenu du président de la République, François Hollande, l'autorisation de consulter toutes les archives relatives à la disparition de son mari. La décision du chef de l'État, tout à fait exceptionnelle, a été suivie d'effet au ministère

\footnotetext{
45 La philosophe Isabelle Delpla en a fait la démonstration dans le cas du massacre de Srebrenica : «Faits, responsabilités, intelligibilité : comparer les enquêtes et les rapports sur Srebrenica », Cultures $\&$ Conflits [En ligne], 65| printemps 2007, mis en ligne le 4janvier 2010, URL: http:// conflits.revues.org/ 2221 [en ligne : consultéle 25 mars 2017].

${ }^{46}$ J ean-Charles Deniau, La Vérité sur la mort de Maurice Audin, Paris, Éditions des Équateurs, 2014, p. 257-265.
} 
Sylvie Thénault, «La disparition de Maurice Audin. Les historiens à l'épreuve d'une enquête impossible (19572004) », Histoire@Politique, n³ 31, janvier-avril 2017 [en ligne, www.histoire-politique.fr]

de la Défense. J osette Audin a reçu copie de documents conservés dans les archives militaires. Elle n'a pas eu à se présenter dans les services d'archives pour consulter les inventaires disponibles et formuler des demandes de consultation de documents dans les règles. Si l'évitement d'une telle procédure repose sur une intention louable, il n'en pose pas moins problème, d'un double point de vue : déontologiquement, en offrant un accès particulier, privilégié, à des documents, mais surtout méthodologiquement. Difficile d'expertiser des documents extraits de fonds épars et de diverses natures et plus encore, de leur donner du sens : rapports, pages de registre, procès-verbaux, fiches...

La quête était en outre incomplète. Il devait y avoir des documents aux Archives nationales à Pierrefitte et aux Archives nationales d'outre-mer (ANOM), à Aix-enProvence, notamment dans les archives judiciaires, en raison de l'instruction de la plainte déposée par J osette Audin. La recherche et la consultation de ces documents suivant cette fois la procédure en vigueur, il fallait orienter J osette Audin au sein des diverses institutions archivistiques, la guider dans la procédure à suivre, l'accompagner dans la consultation en salle de lecture ou dans le décryptage des documents dont des copies lui ont de nouveau été fournies. Une piste a également été suivie, à partir de L'Affaire Audin. Vidal-Naquet y faisait en effet référence à « un dossier sur l'affaire Audin ${ }^{47}$ » constitué par Godard. Le personnel compétent et très coopératif des Archives nationales a pu retrouver, dans le fonds de la Cour de sûreté de l'État, des papiers saisis au domicile de Godard lors des poursuites judiciaires ouvertes contre lui en 1961 - celles qui ont abouti à sa condamnation à mort par contumace. La consultation de ces archives, après obtention d'une dérogation, n’a cependant rien donné.

Cette quête dans les archives est restée vaine, ce qui ne peut être surprenant pour des historiens. Les archives publiques sont en effet les papiers produits par diverses administrations : armée, police, justice, ministères, présidence de la République, etc. Pour y trouver la vérité sur la disparition de Maurice Audin, il aurait fallu que l'un de ces services l'ait connue à l'époque, l'ait mise par écrit, puis que cet écrit ait été conservé et qu'il soit retrouvé aujourd'hui. Il n'était guère plausible que ces conditions soient réunies. De fait, les archives publiques ont enregistré la version mensongère de l'évasion. Se trouvent parmi les archives consultées quantité de documents la concernant, en particulier des documents produits, précisément, par l'élaboration du mensonge : procès-verbaux des militaires et du riverain témoins de la scène, rapport sur la punition du militaire considéré comme responsable, rapport de reconstitution des faits avec schéma des lieux à l'appui, etc. Les pièces du dossier d'instruction elles-mêmes étaient déjà connues de la famille, puisque l'instruction avait été déclenchée, précisément, par J osette Audin. Ses avocats, suivant l'affaire, en avaient déjà eu connaissance. Les archives témoignent par ailleurs de l'activité du Comité Maurice Audin pour la dénonciation de la torture et la recherche de la vérité. D'un point de vue historien, ces archives n'en sont pas moins extrêmement intéressantes, parce qu'elles donnent à voir la Raison d'État en marche. Quantité de documents témoignent du fait que les développements de l'instruction judiciaire, ainsi que l'action du Comité Maurice Audin étaient suivis en très haut lieu. L'éclatement d'une vérité mettant en cause l'armée était crainte et, de ce fait, la recherche de la vérité a été contrariée. Reste que, si les archives devaient un jour apporter du neuf sur ce qui est réellement arrivé à Audin, ce serait certainement de

${ }^{47}$ L'Affaire Audin..., op. cit., p. 163. 
Sylvie Thénault, «La disparition de Maurice Audin. Les historiens à l'épreuve d'une enquête impossible (19572004) », Histoire@Politique, n³1, janvier-avril 2017 [en ligne, www.histoire-politique.fr]

façon incidente. Il est possible d'imaginer qu'un jour un document émerge, contenant un élément nouveau qui, telle une pièce manquante à un puzzle, viendrait conforter l'une ou l'autre des hypothèses envisageables, voire en prouver une au détriment des autres. Une telle découverte est toutefois hasardeuse.

Pour finir, un constat s'impose : bien qu'encore inabouti, le travail d'enquête a été mené. En juin 2014, François Hollande, dans une déclaration officielle, a remis l'affaire entre les mains des historiens, sans reconnaître toute la vérité. Rappelant qu'il avait favorisé les recherches dans les archives pour « lever les incertitudes qui continuent d'entourer les circonstances précises de la mort de M. Audin », le président estimait en effet que « c'est aux historiens qu'il appartient désormais de les préciser ». Et il concluait, au sujet d'Audin : «Il est mort durant sa détention ${ }^{48}$. » François Hollande a ainsi rompu avec la version mensongère de l'évasion, restée la version officielle jusqu'à sa déclaration. Il est cependant évident que l'affirmation selon laquelle Audin « est mort durant sa détention » ne peut satisfaire ceux qui tiennent à la vérité. Qu'il ait été étranglé par l'un de ses tortionnaires, exécuté par un commando spécialisé ou qu'il soit décédé sous la torture, Audin est l'une des victimes de la torture et du système répressif mis en place à Alger en 1957. La responsabilité de l'État, à travers celle de l'armée, est engagée.

\section{L'auteur}

Directrice de recherche CNRS au Centre d'histoire sociale du XXe siècle, Sylvie Thénault est spécialiste du droit et de la répression coloniale en Algérie. Ses travaux portent sur les législations d'exception, les procès, la défense et l'internement des nationalistes pendant la guerre d'indépendance, ainsi que sur l'indigénat dans la longue durée. Elle a notamment publié : Violence ordinaire dans l'Algérie coloniale. Camps, internements, assignations à résidence (Paris, Odile J acob, 2012 ; rééd. Constantine, Média Plus, 2012) ; Histoire de la guerre d'indépendance algérienne (Paris, Flammarion, 2005; rééd. en poche, coll. «Champs », 2012 ; rééd. à TiziOuzou, Maarifa, 2010 ; trad. en arabe, Alger, Dhalab, 2012); Algérie: des "événements" à la guerre. Idées reçues sur la guerre d'indépendance algérienne (Paris, Le Cavalier Bleu, 2012).

\section{Résumé}

Cet article porte sur la contribution des historiens à l'enquête sur la disparition de Maurice Audin à Alger, en 1957, ainsi que sur l'enquête elle-même, qui a rebondi dans les années 2000. Cette disparition est en effet aux sources de l'engagement de Pierre Vidal-Naquet contre la torture. Restée inexpliquée, elle a suscité de nouvelles révélations un demi-siècle plus tard, impliquant cette fois un trio d'enquêteurs: journaliste, documentariste, historienne. Outre les questions épistémologiques soulevées par leur collaboration, l'article analyse les enjeux et les impasses de ces révélations tardives.

Mots clés : guerre d'Algérie; Maurice Audin; disparition forcée; Pierre VidalNaquet ; historiographie ; enquête.

48 Le texte complet de la déclaration est disponible sur le site de l'Élysée : http:// www.elysee.fr/ declarations/article/ message-du-president-de-la-republique-a-l-occasion-de-laremise-du-prix-maurice-audin-pour-les-mathematiques/ [en ligne : consultéle 25 mars 2017]. 
Sylvie Thénault, «La disparition de Maurice Audin. Les historiens à l'épreuve d'une enquête impossible (19572004) », Histoire@Politique, $n^{\circ}$ 31, janvier-avril 2017 [en ligne, www.histoire-politique.fr]

\begin{abstract}
This article addresses the contribution of historians to the investigation of Maurice Audin's disappearance in Algiers in 1957 as well as the investigation itself, which resumed in the 2000s. For this disappearance is at the origin of Pierre Vidal-Naquet's campaign against torture. Still unexplained, it gave rise to new revelations a half century later, this time involving a trio of investigators: a journalist, a documentary filmmaker and a historian. In addition to the epistemological issues raised by their collaboration, the article examines the issues and dead ends presented by these belated revelations.
\end{abstract}

Keywords: Algerian War of Independence; Maurice Audin; Pierre Vidal-Naquet; Historiography; Forced Disappearance.

Pour citer cet article : Sylvie Thénault, « La disparition de Maurice Audin. Les historiens à l'épreuve d'une enquête impossible (1957-2004) », Histoire@Politique, $\mathrm{n}^{\circ} 31$, janvier-avril 2017 [en ligne, www.histoire-politique.fr] 